Hans Föllmer, Alexander Schied

Stochastic Finance

De Gruyter Graduate 


\section{Also of Interest}

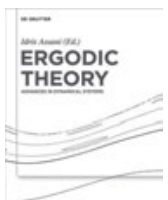

.
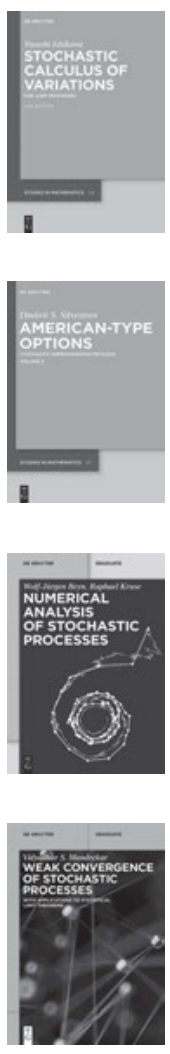

Idris Assani

Ergodic Theory: Advances in Dynamical Systems, 2016

ISBN 978-3-11-046086-5, e-ISBN (PDF) 978-3-11-046151-0, e-ISBN (EPUB) 978-3-11-046091-9

\section{Yasushi Ishikawa}

Stochastic Calculus of Variations: For Jump Processes, 2nd Ed., 2016

ISBN 978-3-11-037776-7, e-ISBN (PDF) 978-3-11-037807-8, e-ISBN (EPUB) 978-3-11-039232-6

Dmitrii S. Silvestrov

American-Type Options: Stochastic Approximation Methods, Volume 2, 2014

ISBN 978-3-11-032968-1, e-ISBN (PDF) 978-3-11-032984-1, e-ISBN (EPUB) 978-3-11-038990-6

Wolf-Jürgen Beyn, Raphael Kruse

Numerical Analysis of Stochastic Processes, 2017

ISBN 978-3-11-044337-0, e-ISBN (PDF) 978-3-11-044338-7, e-ISBN (EPUB) 978-3-11-043555-9

Vidyadhar S. Mandrekar

Weak Convergence of Stochastic Processes: With Applications to Statistical Limit

Theorems, 2016

ISBN 978-3-11-047542-5, e-ISBN (PDF) 978-3-11-047631-6, e-ISBN (EPUB) 978-3-11-047545-6

www.degruyter.com 
Hans Föllmer, Alexander Schied Stochastic Finance

An Introduction in Discrete Time

Fourth revised and extended edition

\section{DE GRUYTER}




\title{
Mathematics Subject Classification 2010
}

Primary: 60-01, 91-01, 91-02; Secondary: 46N10, 60E15, 60G40, 60G42, 91B08, 91B16, 91B30, 91B50, 91B52, 91B70, 91G10, 91G20, 91G80, 91G99.

\author{
Authors \\ Prof. Dr. Hans Föllmer \\ Humboldt-Universität zu Berlin \\ Institut für Mathematik \\ Unter den Linden 6 \\ 10117 Berlin \\ foellmer@math.hu-berlin.de \\ Prof. Dr. Alexander Schied \\ Universität Mannheim \\ Lehrstuhl für Wirtschaftsmathematik \\ A 56 \\ 68159 Mannheim \\ schied@uni-mannheim.de
}

ISBN 978-3-11-046344-6

e-ISBN (PDF) 978-3-11-046345-3

e-ISBN (EPUB) 978-3-11-046346-0

Library of Congress Cataloging-in-Publication Data

A CIP catalog record for this book has been applied for at the Library of Congress.

Bibliographic information published by the Deutsche Nationalbibliothek

The Deutsche Nationalbibliothek lists this publication in the Deutsche Nationalbibliografie;

detailed bibliographic data are available on the Internet at http://dnb.dnb.de.

(C) 2016 Walter de Gruyter GmbH, Berlin/Boston

Typesetting: Compuscript Ltd, Ireland

Printing and binding: $\mathrm{CPI}$ books $\mathrm{GmbH}$, Leck

@ Printed on acid-free paper

Printed in Germany

www.degruyter.com 